02

\title{
A Systematic Exploration of InGaN/GaN Quantum Well-Based Light Emitting Diodes on Semipolar Orientations *
}

\author{
(C) Aparna Das
}

Department of Mathematics and Natural Sciences, College of Sciences and Human Studies, Prince Mohammad Bin Fahd University,

Al Khobar, Kingdom of Saudi Arabia

e-mail: adas@pmu.edu.sa; aparnadasam@gmail.com

Received October 29, 2020

Revised October 29, 2020

Accepted December 08, 2021

Light-emitting diodes (LEDs) based on group III-nitride semiconductors (GaN, AlN, and InN) are crucial elements for solid-state lighting and visible light communication applications. The most widely used growth plane for group III-nitride LEDs is the polar plane (c-plane), which is characterized by the presence of a polarizationinduced internal electric field in heterostructures. It is possible to address long-standing problems in group III-nitride LEDs, by using semipolar and nonpolar orientations of GaN. In addition to the reduction in the polarizationinduced internal electric field, semipolar orientations potentially offer the possibility of higher indium incorporation, which is necessary for the emission of light in the visible range. This is the preferred growth orientation for green/yellow LEDs and lasers. The important properties such as high output power, narrow emission linewidth, robust temperature dependence, large optical polarization ratio, and low-efficiency droop are demonstrated with semipolar LEDs. To harness the advantages of semipolar orientations, comprehensive studies are required. This review presents the recent progress on the development of semipolar InGaN/GaN quantum well LEDs. Semipolar InGaN LED structures on bulk GaN substrates, sapphire substrates, free-standing GaN templates, and on Silicon substrates are discussed including the bright prospects of group III-nitrides.

Keywords: Group III-nitride semiconductor, semipolar, light-emitting diodes, InGaN/GaN quantum well.

\footnotetext{
* Полный текст статьи опубликован в „Optics and Spectroscopy“ 2022 V. 130. № 3.
} 